\title{
A conserved motif of vertebrate $Y$ RNAs essential for chromosomal DNA replication
}

\author{
TIMOTHY J. GARDINER, CHRISTO P. CHRISTOV, ALEXANDER R. LANGLEY, and TORSTEN KRUDE \\ Department of Zoology, University of Cambridge, Cambridge CB2 3EJ, United Kingdom
}

\begin{abstract}
Noncoding Y RNAs are required for the reconstitution of chromosomal DNA replication in late G1 phase template nuclei in a human cell-free system. Y RNA genes are present in all vertebrates and in some isolated nonvertebrates, but the conservation of Y RNA function and key determinants for its function are unknown. Here, we identify a determinant of Y RNA function in DNA replication, which is conserved throughout vertebrate evolution. Vertebrate Y RNAs are able to reconstitute chromosomal DNA replication in the human cell-free DNA replication system, but nonvertebrate $Y$ RNAs are not. A conserved nucleotide sequence motif in the double-stranded stem of vertebrate Y RNAs correlates with Y RNA function. A functional screen of human Y1 RNA mutants identified this conserved motif as an essential determinant for reconstituting DNA replication in vitro. Double-stranded RNA oligonucleotides comprising this RNA motif are sufficient to reconstitute DNA replication, but corresponding DNA or random sequence RNA oligonucleotides are not. In intact cells, wild-type hY1 or the conserved RNA duplex can rescue an inhibition of DNA replication after RNA interference against hY3 RNA. Therefore, we have identified a new RNA motif that is conserved in vertebrate $\mathrm{Y}$ RNA evolution, and essential and sufficient for $\mathrm{Y}$ RNA function in human chromosomal DNA replication.
\end{abstract}

Keywords: noncoding RNA; Y RNA; DNA replication; cell-free system; evolution

\section{INTRODUCTION}

The noncoding Y RNAs were discovered in the early 1980s as an RNA component of Ro ribonucleoprotein particles (Ro RNPs) (Lerner et al. 1981), which also contain Ro60 protein (Chen and Wolin 2004) and other potentially associated proteins. In human cells, four Y RNAs (hY1, hY3, hY4, and hY5 RNA) are transcribed by RNA polymerase III from single copy genes, clustered into a single locus on chromosome 7 (Hendrick et al. 1981; Maraia et al. 1994, 1996; Matera et al. 1995). Y RNAs are not modified and fold into characteristic stem-loop structures (Fig. 1; Farris et al. 1999; Teunissen et al. 2000). The partially complementary $5^{\prime}$ and $3^{\prime}$ domains form a double-stranded stem structure, which is linked by a single-stranded loop domain containing secondary stem-loops. The terminal stem is interrupted by a helical distortion into a lower terminal and an upper central section. The lower stem section and the distorted helical section constitute a conserved

Reprint requests to: Torsten Krude, Department of Zoology, University of Cambridge, Downing Street, Cambridge CB2 3EJ, United Kingdom; e-mail: tk1@mole.bio.cam.ac.uk; fax: +44 (0)1223 336676.

Article published online ahead of print. Article and publication date are at http://www.rnajournal.org/cgi/doi/10.1261/rna.1472009. binding site for the Ro60 protein (Green et al. 1998; Stein et al. 2005). Y RNAs retain a 3' poly(U) tail, which provides a high affinity binding site for the La protein (Wolin and Cedervall 2002). Ro60 and La proteins have been implicated in protecting Y RNAs from exonucleolytic degradation, among other functions (Wolin and Cedervall 2002; Chen and Wolin 2004).

Y RNAs are evolutionarily conserved in vertebrates (Pruijn et al. 1993; Farris et al. 1995; Mosig et al. 2007; Perreault et al. 2007). Homologs of the four Y RNAs have been found in all major classes of vertebrates investigated, but not all of the four Y RNAs are expressed in each vertebrate species (Pruijn et al. 1993; Farris et al. 1995). Two recent computational analyses of genomic data provided new nucleotide sequences for a large number of vertebrate Y RNAs, and allowed a comprehensive insight into the evolution of the vertebrate Y RNA gene cluster (Mosig et al. 2007; Perreault et al. 2007). A common Y RNA gene ancestor split in early gene duplication events into the four extant orthologs, and in some amphibian species, the Y5 genes split further into the $\mathrm{Y} 5$ and $\mathrm{Y} \alpha$ genes. Genomic positions and the orientation of individual Y RNA genes relative to each other are also conserved. In eutheria, however, the locus underwent a partial rearrangement leading 


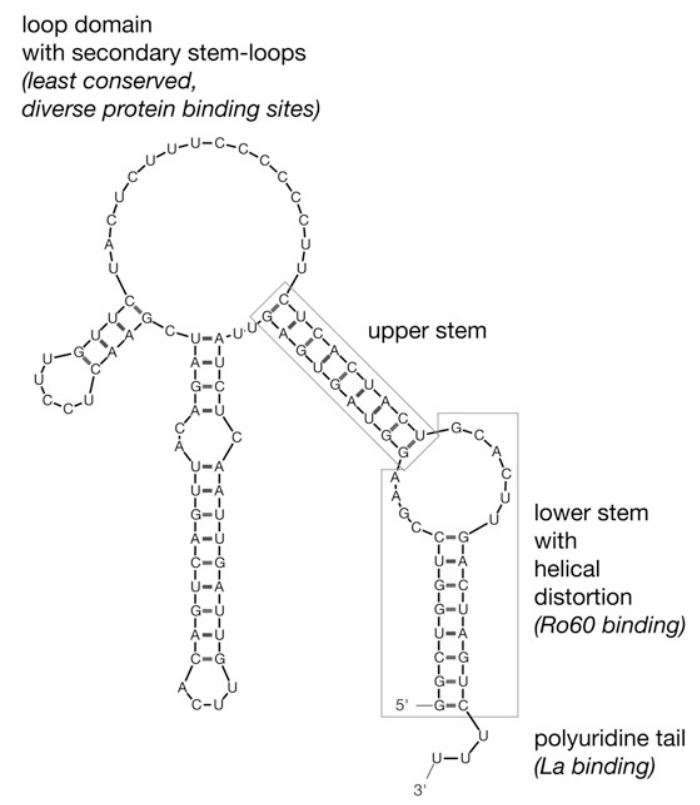

FIGURE 1. Predicted structure of a typical Y RNA and its conserved structural elements. The most stable structure of hY1 RNA was determined by the Mfold algorithm. Locations of conserved structural elements are boxed, with their known functions highlighted in italics.

to a reverse orientation of the Y1 gene relative to the other Y RNA genes. Individual gene-loss events have occurred during the evolution of most classes, resulting in the uneven number of expressed Y RNAs in extant vertebrates. This evolutionary pattern strongly suggests that some key features of vertebrate Y RNA function are conserved between the different Y RNAs, but different features may have evolved into more specialized functions of individual Y RNAs.

Small RNAs with structural features similar to Y RNAs have also been identified through association with orthologs of Ro60 protein in a few isolated nonvertebrate taxa, namely, in the prokaryote Deinococcus radiodurans and the nematode Caenorhabditis elegans (Van Horn et al. 1995; Chen et al. 2000). Genes coding for candidate Y RNAs have also been identified through bioinformatics analyses in the lancelet Branchiostoma floridae (Mosig et al. 2007) and the insect Anopheles gambiae (Perreault et al. 2007). These few $\mathrm{Y}$ RNAs cannot be assigned to any of the four vertebrate $\mathrm{Y}$ RNA clades.

We have recently identified an essential function for human Y RNAs during chromosomal DNA replication (Christov et al. 2006, 2008). In eukaryotes, chromosomal DNA replication is initiated at many replication origins in each cell nucleus (Gilbert 2001; Machida et al. 2005; Costa and Blow 2007). Controlled association of replication initiation proteins with replication origins leads to the formation of DNA replication forks, which replicate the entire genome once, and only once, during the $S$ phase of the cell cycle (Bell and Dutta 2002; Takeda and Dutta 2005; DePamphilis et al. 2006; Arias and Walter 2007). The initi- ation of mammalian chromosomal DNA replication can be reconstituted in cell-free systems, consisting of isolated late G1 phase template nuclei incubated in human cell extracts (Krude 2006). Fractionation and functional reconstitution experiments of a human cell extract have established an essential requirement of hY RNAs for chromosomal DNA replication in the additional presence of protein fractions (Christov et al. 2006). The execution point for Y RNA function has been identified as the initiation stage of DNA replication, and not the chain elongation stage (Krude et al. 2009). A functional requirement of hY RNAs for DNA replication has also been found in cell-based systems as degradation of hY1 or hY3 RNAs in proliferating human cells in culture reduces the proportion of replicating cells in the treated cell population (Christov et al. 2006, 2008). The replication-specific function of hY RNAs is independent of their ability to bind to Ro60 protein as mutational inactivation of the Ro60 binding site of hY1 RNA does not inactivate its activity to promote chromosomal DNA replication (Christov et al. 2006).

In this study, we have investigated the determinant for $\mathrm{Y}$ RNA function and its conservation during evolution. Our experiments have led to the identification of a new motif in the double-stranded upper stem of Y RNAs, which is evolutionarily conserved within vertebrates, but not beyond. This motif is both necessary and sufficient for Y RNA function in chromosomal DNA replication in human cell nuclei.

\section{RESULTS}

\section{Vertebrate Y RNAs can functionally substitute for human $Y$ RNA in chromosomal DNA replication in a cell-free system}

Nucleotide sequences and predicted secondary structures of Y RNAs are conserved within vertebrates (Pruijn et al. 1993; Farris et al. 1995; Mosig et al. 2007; Perreault et al. 2007). We investigated first whether this evolutionary conservation correlates with a possible conservation of their function in chromosomal DNA replication. Y RNAs of representative species from each of the major vertebrate classes were analyzed, using model organisms where available: mouse (Mus musculus, Mammalia; mY1 and mY3 RNA), chicken (Gallus gallus, Aves; cY1 and cY3 RNA), iguana (Iguana iguana, Reptilia; iY3 and iY4 RNA), the South African clawed toad (Xenopus laevis, Amphibia; xY3, $\mathrm{xY} 4, \mathrm{xY} 5$, and $\mathrm{xY} \alpha \mathrm{RNA}$ ) and zebrafish (Danio rerio, Teleostei; zY1 RNA). We generated expression constructs for these vertebrate Y RNAs and synthesized them by in vitro transcription using SP6 RNA polymerase. All these Y RNAs are predicted to fold into the characteristic stemloop structures (Supplemental Fig. S1).

We tested whether the purified vertebrate Y RNAs can initiate chromosomal DNA replication in a human cell-free system. Late G1 phase template nuclei from 
mimosine-arrested human cells initiate semiconservative chromosomal DNA replication in vitro upon incubation in a cytosolic extract from proliferating human cells (Krude 2006). In representative experiments, about $50 \%$ of late G1 phase template nuclei replicate in the presence of the extract, but only $5 \%$ or less in its absence (Krude 2000; Christov et al. 2006). The essential DNA replication proteins are provided by two fractions of the cytosolic extract, termed QA and ArFT (Christov et al. 2006). Addition of fractions QA and ArFT support replication only in about $15 \%$ of the nuclei. This value increases up to $50 \%$ upon addition of any of the four human Y RNAs (Christov et al. 2006).

In the first set of experiments, we used this experimental system to analyze the functional conservation of vertebrate Y RNAs. Human hY1 RNA was used as positive control in each experiment. We normalized the percentages of replicating nuclei to minimize experimental variation between different preparations of template nuclei and extract fractions, setting the percentage of nuclei replicating in unfractionated $\mathrm{S} 100$ extract in each experiment to $100 \%$ (Fig. 2A). In the absence of Y RNA, $33 \% \pm 11 \%$ of template nuclei replicated their DNA (after normalization), and addition of human hY1 RNA increased this value to $75 \% \pm 14 \%$ (Fig. 2A), consistent with our previous data (Christov et al. 2006). Addition of any purified vertebrate Y RNA also increased the proportion of replicating nuclei to $70 \%-90 \%$ (Fig. 2A). This increase of replicating template nuclei by each vertebrate Y RNA over and above the background obtained in the absence of Y RNA was statistically highly significant, and it was not different from the one obtained by hY1 RNA (Supplemental Table 1).

We therefore conclude that vertebrate Y RNAs are able to functionally substitute for hY1 RNAs to initiate chromosomal DNA replication in human template nuclei in vitro.

\section{Nonvertebrate Y RNAs do not support chromosomal DNA replication}

Y RNAs have been identified only in a very few isolated invertebrate and prokaryotic species (Van Horn et al. 1995;
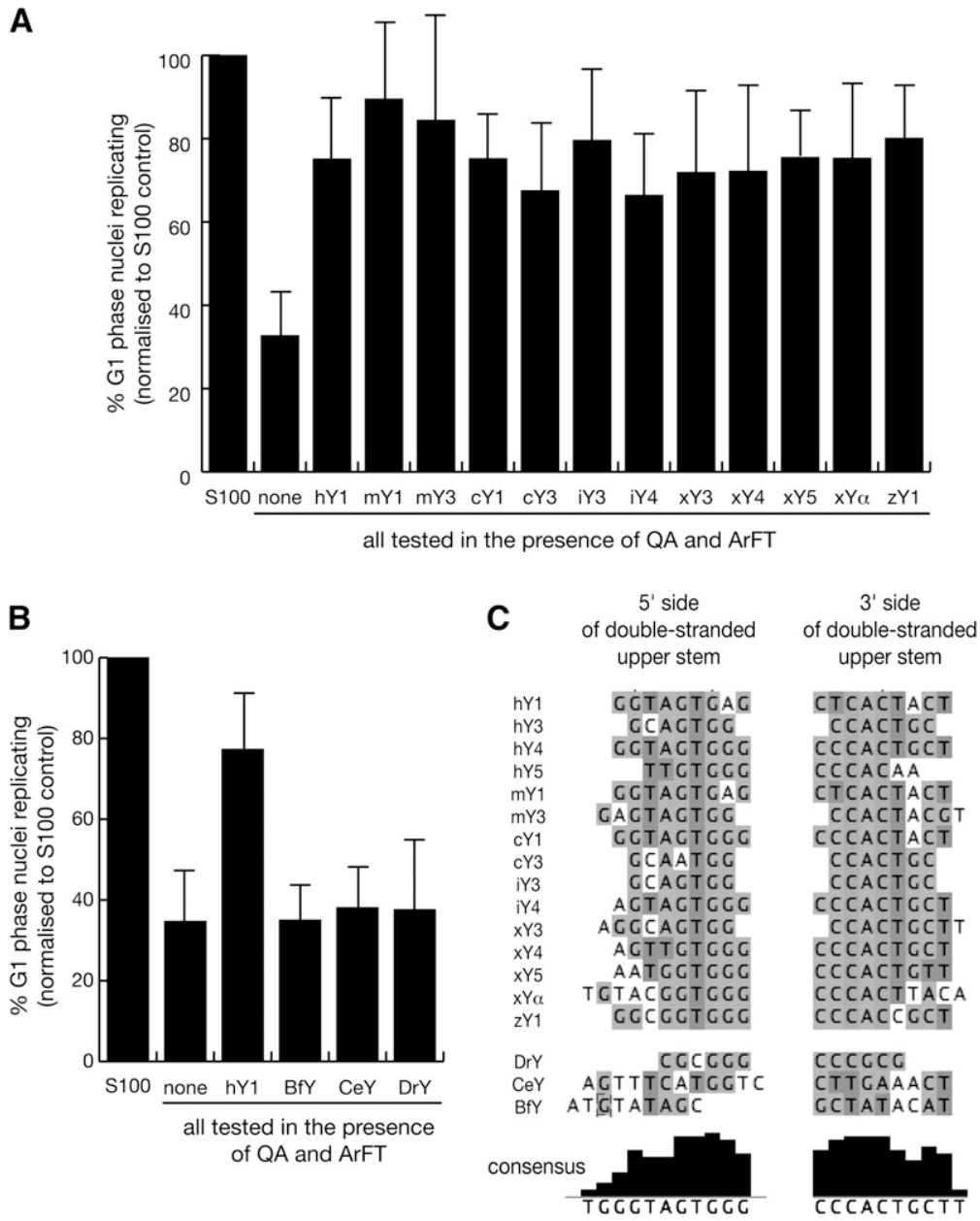

FIGURE 2. Evolutionary conservation of Y RNA function. (A) Vertebrate Y RNAs can substitute for human Y RNAs to initiate chromosomal DNA replication in a human cell-free system. Template nuclei from late G1-phase human cells were incubated with protein fractions QA and ArFT in the presence of $55 \mathrm{nM}$ of the indicated Y RNA. Proportions of replicating nuclei were determined by immunofluorescence microscopy; mean values and standard deviations are shown for each experiment. (B) Nonvertebrate Y RNAs do not support chromosomal DNA replication in this human cell-free system. Nucleotide sequences and predicted structures of these Y RNAs are detailed in Supplemental Figures S1 and S2. Statistical parameters are detailed in Supplemental Table 1. (C) Nucleotide sequence conservation of the upper stem in vertebrate, but not in nonvertebrate Y RNAs. Nucleotide sequences for the $5^{\prime}$ and $3^{\prime}$ sides of the double-stranded upper stem for each vertebrate and nonvertebrate Y RNA were manually selected from the structural predictions made by the Mfold algorithm (see Supplemental Figs. S1,S2). Only sequences of Y RNAs for which functional data are available were considered. These short sequences were aligned by the Kalign algorithm, and displayed in Jalview. Nucleotides are shown in genomic DNA code.

Chen et al. 2000; Mosig et al. 2007; Perreault et al. 2007). These nonvertebrate Y RNAs are predicted to fold into stem-loop structures that bear some similarity to the vertebrate Y RNAs (Supplemental Fig. S2). Based on nucleotide sequence analysis, however, they all fall outside of the vertebrate Y RNA classes (Mosig et al. 2007; Perreault et al. 2007).

In the next set of experiments, we investigated whether nonvertebrate Y RNAs could substitute for human Y1 RNA to initiate chromosomal DNA replication in vitro. 
We generated expression constructs for the Y RNAs from the lancet Branchiostoma floridae (BfY), the nematode Caenorhabditis elegans (CeY), and the prokaryote Deinococcus radiodurans (DrY), synthesized them by in vitro transcription, purified them to homogeneity, and tested whether they could reconstitute chromosomal DNA replication in the human cell-free system (Fig. 2B). Upon addition of any nonvertebrate Y RNA to template nuclei and protein fractions, we did not observe any significant increase in the proportion of replicating nuclei over and above the background seen in the absence of Y RNA (Fig. 2B; Supplemental Table 1).

We therefore conclude that, unlike their vertebrate counterparts, these nonvertebrate Y RNAs are not able to promote the initiation of chromosomal DNA replication in a human cell-free system.

\section{A conserved nucleotide sequence motif in the upper stem of vertebrate $Y$ RNAs correlates with their function in DNA replication}

We carried out nucleotide sequence analysis to identify potential vertebrate Y RNA sequence motifs that would correlate with Y RNA function. The conserved structural features of Y RNAs can be divided into the lower and upper stem, separated by a helical distortion, a poly- $U$ tail at the $3^{\prime}$ end, and the internal loop region containing secondary hairpin structures (highlighted for hY1 RNA in Fig. 1). It has previously been shown that the most conserved nucleotide sequences of the Y RNAs reside in the doublestranded stem region, whereas the nucleotide sequences of the single-stranded loop region are much less conserved (Mosig et al. 2007; Perreault et al. 2007). We have previously shown that the Ro60 binding domain of hY1 RNA, which is located in the lower stem and its helical distortion (Green et al. 1998), can be mutated with no effect on DNA replication (Christov et al. 2006). We therefore focused our analysis of evolutionary conservation on the doublestranded upper stem of the Y RNAs (Fig. 2C). To investigate whether the sequence of the upper stem correlates with Y RNA function, we manually selected the nucleotide sequences from both the $5^{\prime}$ side and $3^{\prime}$ side of the doublestranded upper stem of each tested vertebrate and nonvertebrate Y RNA, as determined by the Mfold algorithm (Supplemental Figs. S1,S2), and aligned these sequences by the Kalign algorithm under default settings (Fig. 2C). This alignment resulted in an imperfect consensus sequence of GUAGUGGG on the 5' strand, and CCCACUGCU on the corresponding $3^{\prime}$ strand of the vertebrate Y RNAs (Fig. 2C). This sequence motif, in particular that of the $3^{\prime}$ strand, was not present in the nonvertebrate Y RNAs (Fig. 2C).

In conclusion, we have identified a new double-stranded nucleotide sequence motif in the upper stem that correlates with the ability of vertebrate Y RNAs to initiate chromosomal DNA replication in human cell-free system. To test whether this motif is directly involved in Y RNA function, we performed a systematic screen of mutant hY1 RNAs.

\section{Deletion of the upper stem of hY1 RNA abrogates $Y$ RNA function in DNA replication}

Initially, we designed a set of deletion mutants of hY1 RNA and asked whether deletion of a particular conserved structural motif of this Y RNA leads to a loss of function in chromosomal DNA replication (Fig. 3). We generated expression constructs, synthesized the mutant RNAs by in vitro transcription, and tested the purified RNAs in the human cell-free chromosomal DNA replication system. The predicted structures of the mutant Y1 RNAs are shown in Figure 3A. Mutants containing deletions of the lower stem $(\Delta \mathrm{LS})$ and internal loop domains $(\Delta \mathrm{LP})$ were as active as the wt hY1 RNA control (Fig. 3B). The $t$-tests showed that the proportion of replicating nuclei was not significantly different between these mutants and the hY1 RNA control (Supplemental Table 2). Similarly, a deletion of the $3^{\prime}$ poly-U tail was also as active as the wt hY1 RNA (data not shown). Deletion of the upper stem ( $\Delta U S)$, however, led to a significant decrease in the proportion of replicating nuclei in this system, down to the level observed without any RNA added (Fig. 3B; Supplemental Table 2). Similarly, deletion of the entire stem region including upper stem, bulge, and lower stem rendered hY1 RNA inactive in supporting the initiation of chromosomal DNA replication in late G1 phase nuclei (data not shown). We therefore conclude that the upper stem of hY1 RNA is essential for $\mathrm{Y}$ RNA function in chromosomal DNA replication in vitro.

\section{Nucleotide sequence elements of Y RNAs are required for their function in DNA replication}

In the next experiment, we determined whether the upper stem domain could confer activity onto an inactive RNA backbone structure. Previously, we have shown that an RNA with a predicted structure identical to hY1 RNA, but of a shuffled nucleotide sequence, does not promote chromosomal DNA replication in the human system (Christov et al. 2006). We therefore designed a pair of two mutant Y RNAs with shuffled nucleotide sequences; $\mathrm{SH}$ and SH:US. SH RNA has a predicted secondary structure similar to wt hY1 RNA, but with a shuffled nucleotide sequence that bears no identity to wt hY1 RNA in any of its domains (Fig. 4A). SH:US RNA has the same sequence as $\mathrm{SH}$, except for the upper stem, which is identical to that of wt hY1 RNA (Fig. 4A). These RNAs were synthesized in vitro, and the purified products were tested in vitro. $\mathrm{SH}$ RNA was not able to promote DNA replication significantly over and above the level obtained without RNA (Fig. 4B; Supplemental Table 2). In contrast, addition of SH:US 
A
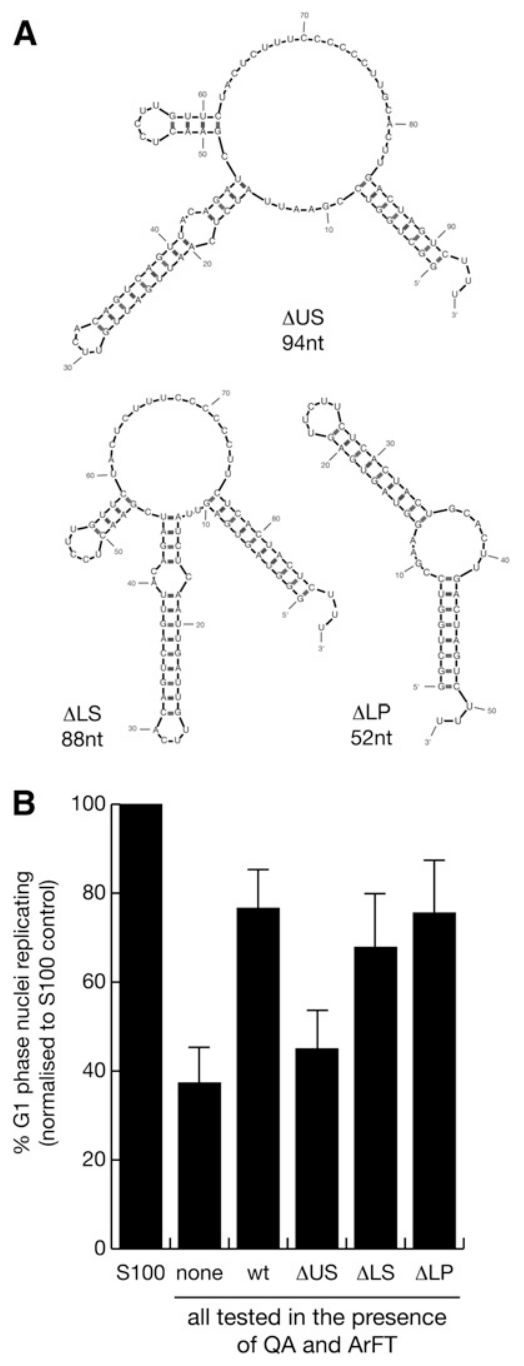

FIGURE 3. The upper stem of hY1 RNA is necessary to reconstitute chromosomal DNA replication in vitro. (A) Nucleotide sequences and predicted secondary structures for mutant hY1 RNAs. Structures are presented as calculated by the Mfold algorithm. $\Delta U S$ is an internal deletion of the upper double-stranded RNA stem, $\Delta \mathrm{LS}$ is a deletion of the lower double-stranded RNA stem, and $\Delta \mathrm{LP}$ is an internal deletion of the single-stranded loop including the secondary stem loops of hY1 RNA. (B) Functional reconstitution of chromosomal DNA replication. Template nuclei from late G1-phase human cells were incubated with protein fractions QA and ArFT in the presence of $55 \mathrm{nM}$ of the indicated mutant hY1 RNAs. Wild-type hY1 RNA is used as a positive control (wt). Proportions of replicating nuclei were determined by immunofluorescence microscopy; mean values and standard deviations are shown for each experiment. Statistical parameters are detailed in Supplemental Table 2.

RNA significantly increased the proportion of replicating nuclei to the level obtained with wt hY1 RNA (Fig. 4B; Supplemental Table 2). We conclude that the nucleotide sequence of the upper stem constitutes an essential motif for Y RNA function in chromosomal DNA replication in vitro.

To investigate further the role of the upper stem of hY1 RNA in DNA replication in vitro, we designed three mu- tant hY1 RNAs with small deletions or base substitutions within the most conserved sequence elements of the upper stem: $\Delta \mathrm{GUG}, \mathrm{ACA}$, and $\Delta \mathrm{U} 18$. The nucleotide sequences of these mutants are shown in Figure 5A. $\Delta$ GUG has a deletion of 3 base pairs (bp) in the center of the upper stem; ACA contains a 3-bp substitution of this region; $\Delta \mathrm{U} 18$ contains a deletion of the highly conserved central $\mathrm{U}$ :A base pair within this motif (Fig. 5A). These mutant hY1 RNAs were synthesized and the purified products tested in vitro. None of these mutant hY RNAs were able to
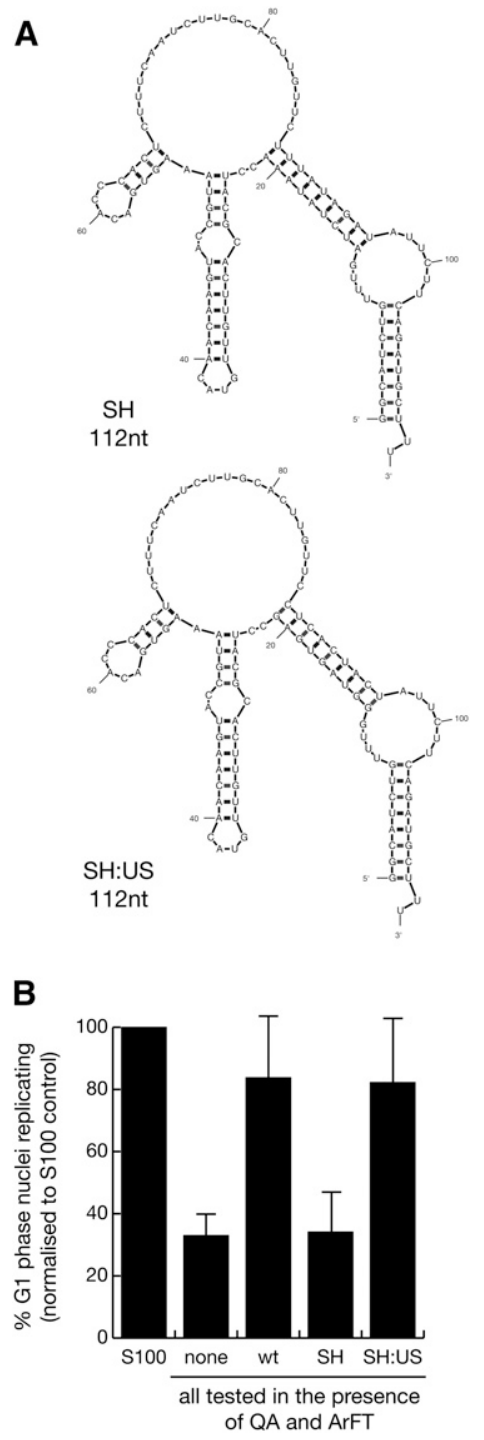

FIGURE 4. Nucleotide sequence elements of the upper stem of hY1 RNA are required to reconstitute chromosomal DNA replication in vitro. (A) Nucleotide sequences and predicted secondary structures for mutant hY1 RNAs. SH has a shuffled nucleotide sequence but maintains the overall predicted structure of hY1 RNA. SH:US contains the nucleotide sequence of the upper stem of hY1 RNA within the context of the SH mutant. (B) Functional reconstitution of chromosomal DNA replication. The indicated mutant Y RNAs were analyzed as detailed in Figure 3. 
A

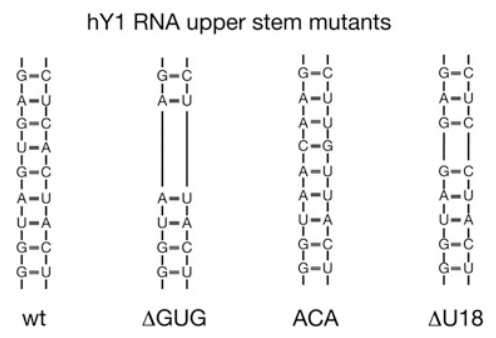

B

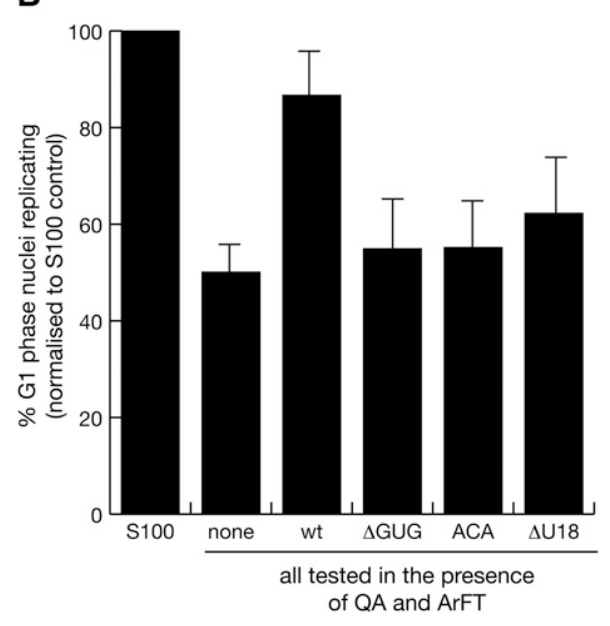

FIGURE 5. A conserved nucleotide sequence element in the upper stem of hY1 RNA is essential to reconstitute chromosomal DNA replication in vitro. (A) Nucleotide sequences of the upper stem of wild-type and mutant hY1 RNAs. $\Delta$ GUG is an internal deletion of the evolutionary conserved vertebrate GUG:CAC 3-bp nucleotide within the double-stranded upper stem, ACA is a replacement of these $3 \mathrm{bp}$ with the sequence ACA:UGU, and $\Delta \mathrm{U} 18$ is an internal deletion of the evolutionary conserved U:A base pair within the upper stem of hY1 RNA. (B) Functional reconstitution of chromosomal DNA replication. The indicated mutant Y RNAs were analyzed as detailed in Figure 3.

promote DNA replication significantly over and above the level obtained without any RNA (Fig. 5B; Supplemental Table 2). We therefore conclude that a specific and evolutionarily conserved nucleotide sequence element around a central U:A base pair within the upper stem of Y RNAs is the key determinant for Y RNA function in chromosomal DNA replication in vitro.

\section{A small double-stranded RNA helix is essential and sufficient for Y RNA function in DNA replication}

To determine whether the upper stem is also sufficient for Y RNA function in vitro, we designed and tested two small mutant hY1 RNAs containing only the sequence of the upper stem: USL and US (Fig. 6A). In one case, the two strands of the upper stem were joined by a small singlestranded pyrimidine loop (USL), while in the other case, the mutant consisted of just a linear double-stranded RNA helix (US). Both of these mutants significantly increased the proportion of replicating nuclei to the same extent as wt hY1 RNA (Fig. 6B; Supplemental Table 2). In contrast, neither the corresponding DNA oligonucleotides nor corresponding random sequence RNAs based on the inactive SH RNA (Fig. 6A) were able to substitute functionally for hY1 RNA (Fig. 6B; Supplemental Table 2). We therefore conclude that the nucleotide sequence-specific RNA motif of the upper double-stranded stem of Y RNAs constitutes the essential and sufficient determinant for their function in chromosomal DNA replication.

A
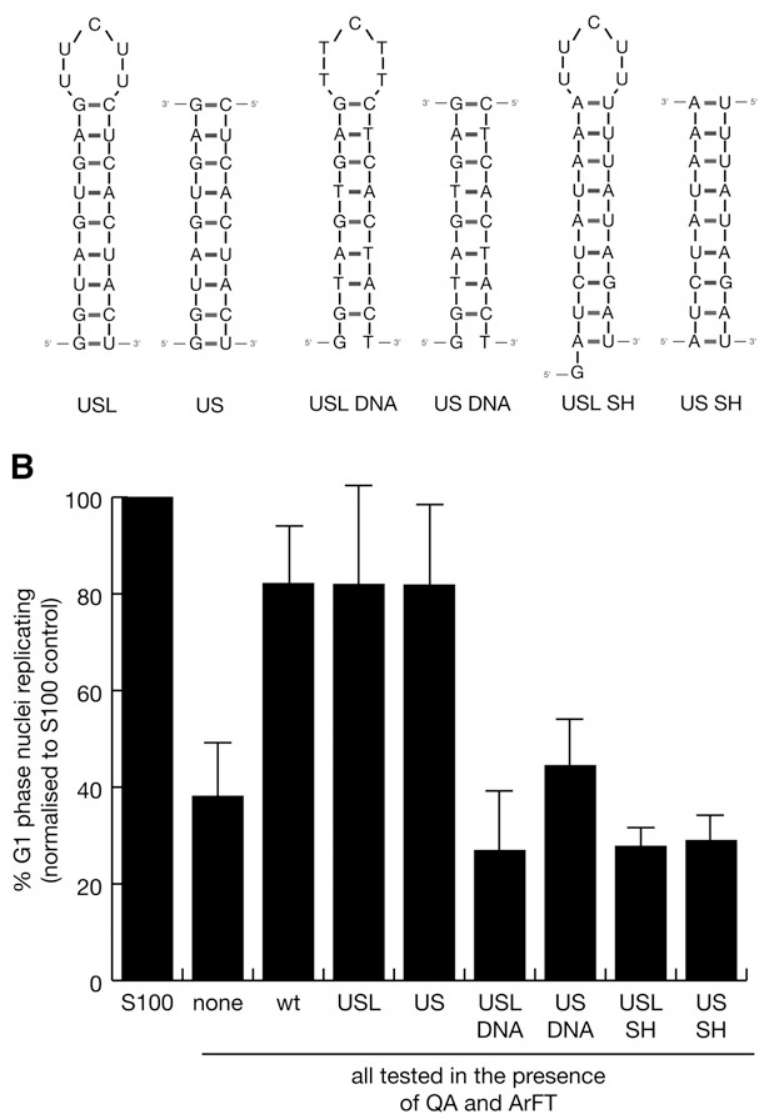

FIGURE 6. A double-stranded RNA helix comprising the upper stem of hY1 RNA is sufficient to reconstitute chromosomal DNA replication in vitro. (A) Nucleotide sequences and predicted secondary structures for RNA and DNA helixes corresponding to the upper stems of hY1 and SH RNAs. USL is the double-stranded RNA helix of the upper stem of hY1 RNA linked by a five-nucleotide singlestranded pyrimidine loop, US is the upper stem as a linear doublestranded RNA helix. USL DNA and US DNA are the corresponding DNA structures. USL SH is the double-stranded RNA helix of the upper stem of SH RNA linked by a five-nucleotide single-stranded pyrimidine loop, US SH is the upper stem as a linear double-stranded RNA helix. Note that USL SH contains an additional $5^{\prime} \mathrm{G}$ residue because of its requirement for in vitro synthesis by SP6 RNA polymerase. (B) Functional reconstitution of chromosomal DNA replication. The indicated RNA and DNA derivatives of hY1 and SH RNAs were analyzed as detailed in Figure 3. 


\section{Exogenous hY1 or USL RNA overcome the inhibition of DNA replication after RNAi against hY3 RNA in vivo}

Finally, we asked whether wt hY1 RNA, or the conserved motif of the upper stem could substitute for hY3 RNA function in proliferating cells in vivo (Fig. 7). We have shown before that RNA interference against hY1 or hY3 RNA in proliferating human cells significantly reduces the proportions of S phase cells (Christov et al. 2006, 2008). Transfection of control siRNA against a nontarget RNA (firefly luciferase mRNA) did not reduce the proportion of $S$ phase cells, and a second transfection $2 \mathrm{~h}$ later of either wt or mutant hY1 RNAs had no significant effect (Fig. 7A). In contrast, RNA interference against hY3 RNA in asynchronously proliferating human cells reduced the proportion of replicating cells by $50 \%$ within $24 \mathrm{~h}$ of transfection (Fig.
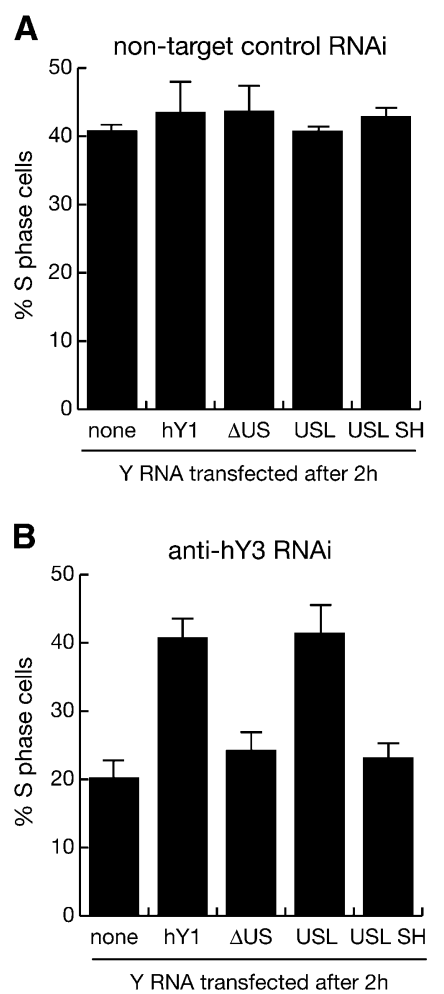

FIGURE 7. The conserved motif of vertebrate $\mathrm{Y}$ RNAs is required chromosomal DNA replication in vivo. $(A)$ Quantification of replicating $\mathrm{S}$ phase cells after control RNA interference (RNAi) against a nontarget RNA, firely luciferase mRNA. (B) Quantification of replicating $\mathrm{S}$ phase cells after RNAi against hY3 RNA. Two hours after transfection of asynchronously proliferating HeLa cells with a control or an hY3 specific siRNA (siRNA hY3c) (Christov et al. 2008), $100 \mathrm{nM}$ of the indicated wild-type and mutant Y RNAs were transfected into the same cells. At $23 \mathrm{~h}$ after RNAi, replicating cells in the population were labeled with BrdU for $1 \mathrm{~h}$. At $24 \mathrm{~h}$, percentages of S phase cells incorporating BrdU into their chromosomal DNA were determined by immunofluorescence microscopy. Mean values and standard deviations are shown for $n=3$ independent experiments. Similar results were obtained with the independent siRNA hY3b (Christov et al. 2008) (data not shown).
7B). This reduction in the proportion of S phase cells was negated when these cells were transfected $2 \mathrm{~h}$ later with either hY1 or USL RNA (Fig. 7B). This negation was statistically significant ( $t$-tests: $P=0.002$ and $P=0.007$ for hY1 and USL RNA, respectively, $n=3$ ). Importantly, transfection with either $\Delta$ US or USL SH RNA, which lack the essential and sufficient domain to support DNA replication in vitro, failed to overcome the inhibition of DNA replication after RNAi against hY3 RNA in vivo (Fig. 7B). We conclude that the conserved double-stranded motif in the upper stem of vertebrate Y RNAs is the key functional determinant for chromosomal DNA replication, both in vitro and in vivo.

\section{DISCUSSION}

In two converging lines of investigation, we have identified a new motif and functional determinant of noncoding $\mathrm{Y}$ RNAs. It consists of double-stranded RNA with an imperfect consensus sequence of GUAGUGGG on the $5^{\prime}$ strand, and CCCACUGCU on the corresponding $3^{\prime}$ strand. This motif is conserved in vertebrate evolution, and it is functionally essential and sufficient to substitute for wild-type (wt) Y RNAs to reconstitute chromosomal DNA replication in human cell-free and cell-based systems. In the first line of investigation, we synthesized a representative range of vertebrate and nonvertebrate Y RNAs and found that all of the vertebrate Y RNAs tested were able to reconstitute the initiation of human chromosomal DNA replication in the presence of human replication proteins, but nonvertebrate Y RNAs were not. A highly conserved short nucleotide sequence motif in the double-stranded upper stem of the vertebrate Y RNAs correlated with their function in DNA replication. In the second line, we carried out a systematic mutagenesis screen of human Y1 RNA and found that the evolutionarily conserved double-stranded region in the upper stem was essential and sufficient to substitute for wild-type Y RNAs to reconstitute DNA replication in vitro and in vivo. These findings have implications for the evolution of Y RNAs as modal functional units and for future experiments to characterize the molecular mechanism by which they regulate the initiation of chromosomal DNA replication in vertebrates.

\section{Evolutionary conservation of Y RNA function in vertebrates}

The genes coding for Y RNAs are evolutionarily conserved in vertebrates (Pruijn et al. 1993; Farris et al. 1995; Mosig et al. 2007; Perreault et al. 2007). An evolutionary history of these genes has been deduced in two recent computational analyses of genomic sequence data (Mosig et al. 2007; Perreault et al. 2007). In this scenario, a common vertebrate $\mathrm{Y}$ RNA ancestor first split into pre-Y1/Y3 and pre-Y4/Y5 genes, which have then split further into the four extant 
monophyletic Y RNA groups, Y1, Y3, Y4, and Y5/Y $\alpha$. During vertebrate evolution, losses of individual Y RNA genes have occurred frequently, so that vertebrate organisms contain between one and four active Y RNA genes (Pruijn et al. 1993; Farris et al. 1995; Mosig et al. 2007; Perreault et al. 2007). Y RNA genes have also been identified in a few isolated nonvertebrates, but systematic searches for Y RNA genes in the genomes of yeasts, plants, echinoderms, urochordates, or the insect Drosophila melanogaster have not been successful (Mosig et al. 2007, and references therein; Perreault et al. 2007). All nonvertebrate Y RNA genes fall outside the evolutionary tree of the four vertebrate Y RNA groups (Mosig et al. 2007; Perreault et al. 2007).

Our data on Y RNA function in chromosomal DNA replication reported here and elsewhere (Christov et al. 2006, 2008) complement the deduced evolutionary history of vertebrate Y RNA genes and provide a possible explanation for the selective pressures on Y RNAs during vertebrate evolution. Within one organism, individual $\mathrm{Y}$ RNAs act redundantly during chromosomal DNA replication in vitro, e.g., degradation of hY1 RNA can be functionally rescued by addition of hY3, hY4, or hY5 RNA (Christov et al. 2006). Importantly, across species boundaries, one vertebrate Y RNA can be functionally substituted by another vertebrate Y RNA, e.g., any vertebrate Y RNA can substitute for human Y RNA to initiate DNA replication in human cell nuclei (this study), or human Y RNAs can substitute for mouse Y RNA to initate chromosomal DNA replication in mouse cell nuclei (Christov et al. 2006; Krude et al. 2009). These functional data establish that the common functional determinant is conserved among vertebrate Y RNAs, but not beyond. A straightforward explanation would be that a role for $\mathrm{Y}$ RNAs in DNA replication has arisen only in ancestral vertebrates and the selective pressure to interact with the highly conserved eukaryotic replication protein machinery caused their subsequent phylogenetic conservation. The presence of conserved Y RNA genes in all vertebrates and the contrasting low number and divergence of nonvertebrate Y RNA genes would support this hypothesis. However, we cannot formally rule out the possibility that nonvertebrate Y RNAs may play a role in nonvertebrate DNA replication. For the prokaryote Deinococcus radiodurans at least, this possibility can be ruled out already as deletion mutants lacking the Y RNA gene are viable and grow with similar kinetics as wt cells (Chen et al. 2007).

We have employed a cell-free system that initiates semiconservative chromosomal DNA replication in somatic mammalian cell nuclei (Krude 2006). Homologous somatic cell-free systems from other nonmammalian vertebrate species have not been developed so far, but a prediction of our results reported here is that mammalian Y RNAs would be able to functionally substitute endogenous $\mathrm{Y}$
RNA in other somatic vertebrate cells. In support of this, we have observed that RNA interference against $x Y$ RNAs in proliferating somatic Xenopus laevis XL177 cells results in a significant reduction of replicating cells in the population (data not shown), similar to RNA interference experiments against hY RNAs performed in proliferating human cells (Christov et al. 2006, 2008). These in vivo data, therefore, further consolidate the conclusion that Y RNA function is conserved in vertebrates.

\section{Functional analysis of hY1 RNA mutants}

In a systematic mutagenesis screen, we have identified that the upper stem of hY1 RNA is essential and sufficient for a reconstitution of chromosomal DNA replication in vitro and in vivo. This analysis therefore confirms the prediction that this domain is a key determinant of Y RNA function, which was drawn from its conservation during vertebrate evolution.

Deletions of the lower stem of hY1 RNA were fully able to initiate DNA replication in vitro, showing that this conserved domain is dispensable for this function. Previously, we have shown that small internal deletions and base substitution mutants of the Ro60 binding domain, located in the lower stem of vertebrate Y RNAs (Green et al. 1998; Stein et al. 2005) that prevent Ro60 from binding, are as active in reconstituting DNA replication as wt hY1 RNA (Christov et al. 2006). Here, we extend this observation by showing that a hY1 mutant with a deletion of the entire lower stem also supports DNA replication as efficiently as the wt hY1 RNA. The binding site for Ro60 is highly conserved in all vertebrate and nonvertebrate Y RNAs; in fact, the presence of a binding site for Ro60 and its orthologs is routinely used to classify a small RNA as a "Y RNA." These data suggest that the binding site for Ro60 and the functional motif required for DNA replication are independent units. Therefore, vertebrate Y RNAs can be considered as functional modular structures.

As all known Y RNAs contain binding sites for Ro60, it is therefore possible that Ro60 performs an important cellular function involving Y RNAs. Functions for Ro60 have been implicated in cellular responses to cytotoxic stress (Labbe et al. 2000; Chen et al. 2003), in a quality control for small RNAs (Chen and Wolin 2004; Stein et al. 2005; Fuchs et al. 2006; Chen et al. 2007; Hogg and Collins 2007), the export of associated RNAs from the nucleus (Simons et al. 1996), and the protection of associated RNAs from exonucleolytic degradation (Chen and Wolin 2004). In vertebrates, the binding site for Ro60 is adjacent to the domain essential for DNA replication, and thus insulates it from the $5^{\prime}$ and $3^{\prime}$ ends of the RNA. In light of our data, it is therefore tempting to speculate that although Ro60 is dispensable for DNA replication (Chen et al. 2003; Xue et al. 2003; Christov et al. 2006), it may nonetheless be involved in DNA replication indirectly, either by protecting or insulating 
the replication domain from exonucleases, or by regulating the intracellular localization of Y RNAs.

Internal deletion of the less conserved central singlestranded loop domain of hY1 RNA and its secondary stemloops did not inactivate the replication-promoting function of this RNA. Several proteins and RNPs have been reported to interact with this domain in different Y RNAs, including nucleolin, hnRNP K, hnRNP I, and 5S RNP (Fabini et al. 2001; Fouraux et al. 2002; Hogg and Collins 2007). These interactions do not occur with all Y RNAs (Fabini et al. 2000, 2001; Fouraux et al. 2002). This heterogeneity points toward additional specialized functions of individual $\mathrm{Y}$ RNAs mediated by this domain, which do not play a role in the cell-free DNA replication system. The central domain of Y RNAs may therefore just serve the minimal purpose of providing a hinge function to allow efficient annealing of the $5^{\prime}$ and $3^{\prime}$ domains into the stem structure that comprises the essential DNA replication domain and the Ro60 binding site. Reduced selective pressure on this central domain in vertebrate evolution would therefore have led to its diversification and acquisition of functions such as RNA quality control surveillance (Hogg and Collins 2007, 2008).

Deletion of the upper stem, or small internal deletions and base substitutions within the upper stem of hY1 RNA resulted in inactive $\mathrm{Y}$ RNA mutants in vitro and in vivo, establishing that this domain constitutes the key functional determinant for Y RNA function in chromosomal DNA replication. In fact, transplantation of this domain into an inert scaffold RNA structure led to the generation of a replication-promoting novel RNA in vitro. Key elements of this double-stranded domain of vertebrate Y RNAs are specific short nucleotide sequence elements on both strands and their biochemical identity as RNA, not DNA. These features imply that this domain could serve as a specific binding site for an interacting protein or protein complex. However, no proteins that specifically interact with this domain have been described to date. We are currently isolating and identifying cellular proteins that interact with wild-type human Y RNAs and their mutants to identify specific Y RNA-protein interactions that are relevant for the function of Y RNAs in chromosomal DNA replication. Results of this separate study will be reported elsewhere.

Finally, our data are not inconsistent with a role of $\mathrm{Y}$ RNAs as substrates for endonucleolytic processing of the primary Y RNA transcript into shorter active components. Such a hypothetical processing would be analogous to the processing of miRNA precursors into active miRNAs by the RNase III endonucleases Drosha and Dicer (Bartel 2004; $\mathrm{Du}$ and Zamore 2005). We have shown here that a short linear double-stranded RNA fragment derived from hY1 RNA is sufficient to reconstitute the initiation of chromosomal DNA replication in vitro, whereas a random sequence double-stranded RNA of the same length is not. We would therefore predict that an endonucleolytically processed product of a primary vertebrate Y RNA transcript encompassing this domain would also be active in initiating DNA replication. Preliminary experiments in our lab indicated that a fraction of Y RNAs is indeed cleaved endonucleolytically during the DNA replication in vitro (data not shown). It is therefore tempting to speculate that $\mathrm{Y}$ RNAs may be genuine substrates for RNase III type endonucleases.

Our demonstration that the truncated mutant USL RNA can functionally substitute for wt Y RNA in vitro, and in vivo also provides an explanation for the observation by Northern blotting that full-length mouse Y RNA levels are reduced in Ro60 knockout cells without an apparent inhibition of DNA replication (Chen et al. 2003; Xue et al. 2003). Provided that Ro60 depletion leads to increased nucleolytic degradation of Y RNAs (Chen et al. 2003; Xue et al. 2003), any resulting fragments containing only the 9-bp upper stem RNA duplex would still be functionally active and available to support chromosomal DNA replication, as seen in our RNAi rescue experiments.

In conclusion, our biochemical and cell biological data described here have led to the identification of a novel domain of vertebrate Y RNAs, which is essential and sufficient for Y RNA function during chromosomal DNA replication. This finding offers a functional explanation for the conservation of Y RNA genes during vertebrate evolution.

\section{MATERIALS AND METHODS}

\section{DNA replication reactions and analysis}

Cell culture, cell synchronization, preparation of template nuclei, and analysis of DNA replication reactions were performed as described (Krude 2000; Szüts et al. 2003, 2005, and references therein). Template nuclei were prepared from human HeLa cervix carcinoma and EJ30 bladder carcinoma cells. Cell extracts were prepared from asynchronously proliferating HeLa cells, and fractionation of the cytosolic extract was carried out exactly as described (Christov et al. 2006). Recombinant Y RNA was used in reconstituted DNA replication reactions at a concentration of $55 \mathrm{nM}$ as described (Christov et al. 2006).

Raw percentages of replicating nuclei were normalized for each independent experiment to minimize systematic errors. Percentages of replicating nuclei in the presence of unfractionated S100 cytosolic extract were set as $100 \%$, and raw percentages obtained in reconstitution experiments were determined as a fraction thereof. Means and standard deviations were calculated from normalized data. The $t$-tests (two-tailed, unpaired, unequal variance) were performed on normalized data.

\section{Expression of recombinant Y RNAs}

Sequences for vertebrate and nonvertebrate Y RNA genes have been published (Farris et al. 1995; Mosig et al. 2007; Perreault et al. 2007, and references therein). Full-length cDNA sequences encoding for vertebrate and nonvertebrate $\mathrm{Y}$ RNAs and for mutant hY1 RNAs were generated by PCR amplification using 
synthetic DNA templates (Sigma-Genosys). All recombinant cDNA sequences contained a 5' SP6 promoter site as previously described (Christov et al. 2006). The PCR products were cloned using the Topo-TA 2.1 cloning kit (Invitrogen) as previously described (Christov et al. 2006). Recombinant plasmid templates were confirmed by DNA sequencing. Expression templates containing the SP6 promoter were obtained by PCR amplification of the recombinant plasmids to produce linear templates with a runoff termination site at the native $3^{\prime}$ ends. USL and USL SH RNAs were expressed from synthetic DNA templates (Sigma-Genosys).

Recombinant Y RNAs were synthesized by in vitro transcription using SP6 RNA polymerase. The size and purity of all in vitro synthesized RNA was confirmed using $8 \mathrm{M}$ urea denaturing polyacylamide gel electrophoresis and staining with SYBR Gold Invitrogen), as previously described (Christov et al. 2006; data not shown). DNA oligonucleotides were obtained from a commercial source (Sigma Genosys).

\section{RNA interference in vivo}

Individual siRNAs were chemically synthesized using an Ambion Silencer siRNA construction kit from primer pair sequences as detailed previously (Christov et al. 2008). Transfections were performed with $10 \mathrm{nM}$ siRNAs using Lipofectamine 2000 reagent (Invitrogen) and OptiMEM (GibcoBRL), as specified by the supplier. Two hours after this transfection, $100 \mathrm{nM}$ of hY1 RNA or the specified mutant RNAs were transfected into the same cell populations. Identical concentrations of Lipofectamine were used for all transfections.

The $\mathrm{S}$ phase index was determined at $24 \mathrm{~h}$ after siRNA transfection by pulse-labeling of replication foci with BrdUTP for $1 \mathrm{~h}$ prior to preparation of the nuclei. Detection of BrdU incorporation and determination of the percentages of S phase cells incorporating BrdU were determined by standard immunofluorescence microscopy.

\section{Bioinformatics}

Predicted secondary structures for all RNAs were calculated from the full-length nucleotide sequence using the Mfold v3.2 RNA folding algorithm (web server at: http://frontend.bioinfo.rpi.edu/ applications/mfold/cgi-bin/rna-form1.cgi) under default conditions (Mathews et al. 1999; Zuker 2003). Outputs were converted into pdf file format. Multiple nucleotide sequence alignments were carried out using the K-align alignment algorithm (web server at: http://www.ebi.ac.uk/kalign/) under default conditions (Lassmann and Sonnhammer 2005). The readout was visualized using Jalview v2.x (http://www.jalview.org) (Clamp et al. 2004).

\section{SUPPLEMENTAL MATERIAL}

Supplemental material can be found at http://www.rnajournal.org.

\section{ACKNOWLEDGMENTS}

We thank Drs. Cath Green and Giselle Walker for critical reading of the manuscript. This work was supported by Cancer Research UK (project grants C1471/A5993 and C1471/A8448). T.J.G. and A.R.L. were supported by Research Studentships from the Medical Research Council (MRC).
Received November 19, 2008; accepted April 2, 2009.

\section{REFERENCES}

Arias EE, Walter JC. 2007. Strength in numbers: Preventing rereplication via multiple mechanisms in eukaryotic cells. Genes \& Dev 21: 497-518.

Bartel DP. 2004. MicroRNAs: Genomics, biogenesis, mechanism, and function. Cell 116: 281-297.

Bell SP, Dutta A. 2002. DNA replication in eukaryotic cells. Annu Rev Biochem 71: 333-374.

Chen X, Wolin SL. 2004. The Ro $60 \mathrm{kDa}$ autoantigen: Insights into cellular function and role in autoimmunity. J Mol Med 82: 232-239.

Chen X, Quinn AM, Wolin SL. 2000. Ro ribonucleoproteins contribute to the resistance of Deinococcus radiodurans to ultraviolet irradiation. Genes \& Dev 14: 777-782.

Chen X, Smith JD, Shi H, Yang DD, Flavell RA, Wolin SL. 2003. The Ro autoantigen binds misfolded U2 small nuclear RNAs and assists mammalian cell survival after UV irradiation. Curr Biol 13: 2206-2211.

Chen X, Wurtmann EJ, Van Batavia J, Zybailov B, Washburn MP, Wolin SL. 2007. An ortholog of the Ro autoantigen functions in $23 \mathrm{~S}$ rRNA maturation in D. radiodurans. Genes \& Dev 21: 1328-1339.

Christov CP, Gardiner TJ, Szüts D, Krude T. 2006. Functional requirement of noncoding Y RNAs for human chromosomal DNA replication. Mol Cell Biol 26: 6993-7004.

Christov CP, Trivier E, Krude T. 2008. Noncoding human Y RNAs are overexpressed in tumours and required for cell proliferation. $\mathrm{Br} \mathrm{J}$ Cancer 98: 981-988.

Clamp M, Cuff J, Searle SM, Barton GJ. 2004. The Jalview Java alignment editor. Bioinformatics 20: $426-427$.

Costa S, Blow JJ. 2007. The elusive determinants of replication origins. EMBO Rep 8: 332-334.

DePamphilis ML, Blow JJ, Ghosh S, Saha T, Noguchi K, Vassilev A. 2006. Regulating the licensing of DNA replication origins in metazoa. Curr Opin Cell Biol 18: 231-239.

Du T, Zamore PD. 2005. microPrimer: The biogenesis and function of microRNA. Development 132: 4645-4652.

Fabini G, Rutjes SA, Zimmermann C, Pruijn GJ, Steiner G. 2000. Analysis of the molecular composition of Ro ribonucleoprotein complexes. Identification of novel Y RNA-binding proteins. Eur J Biochem 267: 2778-2789.

Fabini G, Raijmakers R, Hayer S, Fouraux MA, Pruijn GJ, Steiner G. 2001. The heterogeneous nuclear ribonucleoproteins I and $\mathrm{K}$ interact with a subset of the ro ribonucleoprotein-associated $\mathrm{Y}$ RNAs in vitro and in vivo. J Biol Chem 276: 20711-20718.

Farris AD, Koelsch G, Pruijn GJ, van Venrooij WJ, Harley JB. 1999. Conserved features of Y RNAs revealed by automated phylogenetic secondary structure analysis. Nucleic Acids Res 27: 1070-1078.

Farris AD, O'Brien CA, Harley JB. 1995. Y3 is the most conserved small RNA component of Ro ribonucleoprotein complexes in vertebrate species. Gene 154: 193-198.

Fouraux MA, Bouvet P, Verkaart S, van Venrooij WJ, Pruijn GJ. 2002. Nucleolin associates with a subset of the human Ro ribonucleoprotein complexes. J Mol Biol 320: 475-488.

Fuchs G, Stein AJ, Fu C, Reinisch KM, Wolin SL. 2006. Structural and biochemical basis for misfolded RNA recognition by the Ro autoantigen. Nat Struct Mol Biol 13: 1002-1009.

Gilbert DM. 2001. Making sense of eukaryotic DNA replication origins. Science 294: 96-100.

Green CD, Long KS, Shi H, Wolin SL. 1998. Binding of the 60-kDa Ro autoantigen to Y RNAs: Evidence for recognition in the major groove of a conserved helix. RNA 4: 750-765.

Hendrick JP, Wolin SL, Rinke J, Lerner MR, Steitz JA. 1981. Ro small cytoplasmic ribonucleoproteins are a subclass of La ribonucleoproteins: Further characterization of the Ro and La small ribonucleoproteins from uninfected mammalian cells. Mol Cell Biol 1: 1138-1149. 
Hogg JR, Collins K. 2007. Human Y5 RNA specializes a Ro ribonucleoprotein for 5S ribosomal RNA quality control. Genes \& Dev 21: 3067-3072.

Hogg JR, Collins K. 2008. Structured noncoding RNAs and the RNP Renaissance. Curr Opin Chem Biol 12: 684-689.

Krude T. 2000. Initiation of human DNA replication in vitro using nuclei from cells arrested at an initiation-competent state. J Biol Chem 275: 13699-13707.

Krude T. 2006. Initiation of chromosomal DNA replication in mammalian cell-free systems. Cell Cycle 5: 2115-2122.

Krude T, Christov CP, Hyrien O, Marheineke K. 2009. Y RNA functions at the initiation step of mammalian chromosomal DNA replication. J Cell Sci 122 (in press).

Labbe JC, Burgess J, Rokeach LA, Hekimi S. 2000. ROP-1, an RNA quality-control pathway component, affects Caenorhabditis elegans dauer formation. Proc Natl Acad Sci 97: 13233-13238.

Lassmann T, Sonnhammer EL. 2005. Kalign-An accurate and fast multiple sequence alignment algorithm. BMC Bioinformatics 6: 298 .

Lerner MR, Boyle JA, Hardin JA, Steitz JA. 1981. Two novel classes of small ribonucleoproteins detected by antibodies associated with lupus erythematosus. Science 211: 400-402.

Machida YJ, Hamlin JL, Dutta A. 2005. Right place, right time, and only once: Replication initiation in metazoans. Cell 123: 13-24.

Maraia RJ, Sasaki-Tozawa N, Driscoll CT, Green ED, Darlington GJ. 1994. The human Y4 small cytoplasmic RNA gene is controlled by upstream elements and resides on chromosome 7 with all other hY scRNA genes. Nucleic Acids Res 22: 3045-3052.

Maraia R, Sakulich AL, Brinkmann E, Green ED. 1996. Gene encoding human Ro-associated autoantigen Y5 RNA. Nucleic Acids Res 24: 3552-3559.

Matera AG, Frey MR, Margelot K, Wolin SL. 1995. A perinucleolar compartment contains several RNA polymerase III transcripts as well as the polypyrimidine tract-binding protein, hnRNP I. J Cell Biol 129: 1181-1193.

Mathews DH, Sabina J, Zuker M, Turner DH. 1999. Expanded sequence dependence of thermodynamic parameters improves prediction of RNA secondary structure. J Mol Biol 288: 911-940.
Mosig A, Guofeng M, Stadler BMR, Stadler PF. 2007. Evolution of the vertebrate Y RNA cluster. Theory Biosci 129: 9-14.

Perreault J, Perreault JP, Boire G. 2007. The Ro Associated Y RNAs in Metazoans: Evolution and diversification. Mol Biol Evol 24: 16781689.

Pruijn GJ, Wingens PA, Peters SL, Thijssen JP, van Venrooij WJ. 1993. Ro RNP associated Y RNAs are highly conserved among mammals. Biochim Biophys Acta 1216: 395-401.

Simons FH, Rutjes SA, van Venrooij WJ, Pruijn GJ. 1996. The interactions with Ro60 and La differentially affect nuclear export of hY1 RNA. RNA 2: 264-273.

Stein AJ, Fuchs G, Fu C, Wolin SL, Reinisch KM. 2005. Structural insights into RNA quality control: The Ro autoantigen binds misfolded RNAs via its central cavity. Cell 121: 529-539.

Szüts D, Kitching L, Christov C, Budd A, Peak-Chew S, Krude T. 2003. RPA is an initiation factor for human chromosomal DNA replication. Nucleic Acids Res 31: 1725-1734.

Szüts D, Christov C, Kitching L, Krude T. 2005. Distinct populations of human PCNA are required for initiation of chromosomal DNA replication and concurrent DNA repair. Exp Cell Res 311: 240-250.

Takeda DY, Dutta A. 2005. DNA replication and progression through S phase. Oncogene 24: 2827-2843.

Teunissen SW, Kruithof MJ, Farris AD, Harley JB, Venrooij WJ, Pruijn GJ. 2000. Conserved features of Y RNAs: A comparison of experimentally derived secondary structures. Nucleic Acids Res 28: 610-619.

Van Horn DJ, Eisenberg D, O’Brien CA, Wolin SL. 1995. Caenorhabditis elegans embryos contain only one major species of Ro RNP. RNA 1: 293-303.

Wolin SL, Cedervall T. 2002. The La protein. Annu Rev Biochem 71: 375-403.

Xue D, Shi H, Smith JD, Chen X, Noe DA, Cedervall T, Yang DD, Eynon E, Brash DE, Kashgarian M, et al. 2003. A lupus-like syndrome develops in mice lacking the Ro $60-\mathrm{kDa}$ protein, a major lupus autoantigen. Proc Natl Acad Sci 100: 7503-7508.

Zuker M. 2003. Mfold web server for nucleic acid folding and hybridization prediction. Nucleic Acids Res 31: 3406-3415. 

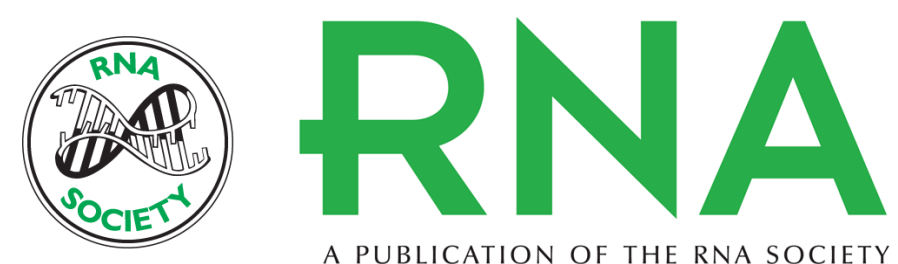

A PUBLICATION OF THE RNA SOCIETY

\section{A conserved motif of vertebrate Y RNAs essential for chromosomal DNA replication}

Timothy J. Gardiner, Christo P. Christov, Alexander R. Langley, et al.

RNA 2009 15: 1375-1385 originally published online May 27, 2009

Access the most recent version at doi:10.1261/rna.1472009

\section{Supplemental http://rnajournal.cshlp.org/content/suppl/2009/05/28/rna.1472009.DC1 \\ Material}

References This article cites 47 articles, 17 of which can be accessed free at: http://rnajournal.cshlp.org/content/15/7/1375.full.html\#ref-list-1

\section{License}

Email Alerting Receive free email alerts when new articles cite this article - sign up in the box at the Service top right corner of the article or click here.

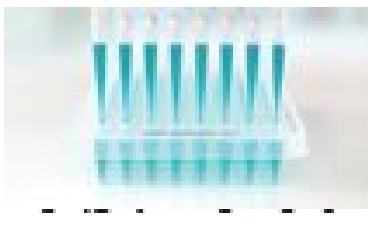

Providing Precise Solutions for your research.

To subscribe to RNA go to:

http://rnajournal.cshlp.org/subscriptions 